\title{
JOURNAL OF HEALTH
}

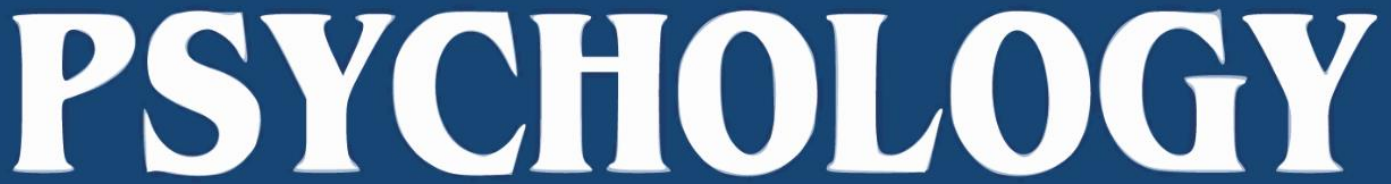

An Interdisciplinary, International Journal

\section{VOLUME 25 - NUMBER 2 aEBRUARY 2020}

Soltani, P., Figueiredo, P., \& Vilas-Boas, J. P. (2020). Does exergaming drive future physical activity and sport intentions? Journal of Health Psychology.

doi:10.1177/1359105320909866 


\title{
Does exergaming drive future physical activity and sport intentions?
}

\author{
Pooya Soltani ${ }^{1,2,3,4}$, Pedro Figueiredo ${ }^{5,6}$, João Paulo Vilas-Boas ${ }^{1}$ \\ ${ }^{1}$ Centre of Research, Education, Innovation and Intervention in Sport (CIFI'D), Faculty of Sport, Porto \\ Biomechanics Laboratory (LABIOMEP), University of Porto, Rua Dr. Plácido Costa, 91, 4200-450 \\ Porto, Portugal. \\ ${ }^{2}$ Etienne-Jules Marey Institute of Movement Sciences, Faculty of Sport Sciences, Aix-Marseille \\ University, 163 Avenue de Luminy, 13009 Marseille, France. \\ ${ }^{3}$ Assistive Technologies Innovation Centre (ATiC), University of Wales Trinity Saint David, Technium 1, \\ Kings Road, Swansea SA1 8PH, United Kingdom. \\ ${ }^{4}$ Department of Physical Education and Sport Sciences, School of Education and Psychology, Shiraz \\ University, Pardis-e-Eram, Eram Square, 71946-84759 Shiraz, Iran. \\ ${ }^{5}$ Portuguese Football School, Portuguese Football Federation, Avenida das Seleções, 1495-433 Oeiras, \\ Portugal. \\ ${ }^{6}$ Research Center in Sports Sciences, Health Sciences and Human Development, CIDESD, University \\ Institute of Maia, ISMAI, Avenida Carlos de Oliveira Campos, Castêlo da Maia, 4475-690 Maia, \\ Portugal.
}

\section{Abstract}

We studied how usability and playability of sport exergames affect future intentions of participation in physical activity (PA) or actual sport. We employed questionnaires to measure participants' enjoyment, usability, game-experience, and future intentions of PA and real sport. We compared the outcomes based on players' gender, previous realswimming, and exergame experience. Psychological parameters were not different between groups but players without exergame experience enjoyed the game more. PA intentions increased for all participants but not swimming intentions. The limitations of current gaming systems and their effects on players' gaming experience and intentions are discussed.

Keywords: Enjoyment; Game experience; System usability; Exercise; Intention 


\section{Introduction}

Researchers have suggested that screen time and overall sedentary behaviors increase through adolescence (Pearson et al., 2017; Straatmann et al., 2019). Computer and video game use prompt significant levels of sedentary behavior (Arundell et al., 2019). As technology is advancing and becoming more accessible, it is important to understand how it can promote innovative physical activity (PA) interventions (Lewis et al., 2017). Given the popularity of video games, active video games (exergames) appear to be an ideal platform to combat sedentary video gaming and to administer PA interventions. By affecting some of the key mediators of PA behavior change, exergames may also offer affordable, safe, and controlled experiences that could be played at home (Rhodes et al., 2018). These games can also overcome barriers to PA such as weather, neighborhood safety, and socioeconomic factors. Some exergames may elicit high PA levels and adherence (Staiano et al., 2018), and players find virtual versions of traditional exercises more enjoyable (Farrow et al., 2018; Moholdt et al., 2017). Additionally, because they incorporate body movements into gaming scenarios, the new skills acquired while exergaming might also be transferable to other activities (Staiano and Calvert, 2011; Bronner et al., 2016; Benzing and Schmidt, 2018). For example, Fery and Ponserre (2001) found that golfers could control their putting force to a greater extent following the performance of a similar task using exergames.

Despite positive short-term results, exergames may show little effectiveness over a prolonged period of time (Schwarz et al., 2018; Biddiss and Irwin, 2010). Exergames are generally less enjoyable than sedentary video games and may be played less frequently over time, possibly due to players' rapid mastery of the mechanics underlying such games (Soltani et al., 2017; Lyons et al., 2011). Additional research demonstrates that after three months of home exergaming, most physiological parameters remain unchanged (Owens et al., 2011). Furthermore, exergames may engender lower motivational outcomes when compared to traditional methods of exercising (Wu et al., 2015). Additionally, traditional human-led exercises produce more vigorous-intensity PA compared to their respective virtual counterparts (Schneekloth and Brown, 2018). The lower amounts of physical and cognitive fidelity of exergames may not lead to the positive transfer of learned skills (Soltani et al., 2016). Moreover, skills learned using sport exergames may lead to poor motor habits that could be difficult to rectify (Pedersen et al., 2017). There is also limited research on whether being active using exergames might influence players to participate in more PA after exposure to gameplay. Baranowski (2017) suggested that the low-intensity PA during exergaming did not transfer to the free play period post-intervention in a sample of children. However, Carson et al. (2015) showed that involving adolescents in screen-based PA could reduce screen time post-intervention.

With such contradictory results and barriers in sustained exergame engagement (Dixon et al., 2010), there is a distinct need to explore whether playing exergames can influence players' intentions for participating in PA and real sports. In game-like interventions, the transfer to PA is regarded as a desirable outcome rather than a design requirement (Kuipers et al., 2017). In order to change intentions, we should understand 1) how different players (with different genders, gaming background, and sports background) perceive the games, and 2) how specific game features might change players' intentions. 


\section{Gender, exergame experience, and real-sport experience}

Male and female players might have similar light- and moderate-to-vigorous PA behavior during exergaming (Quan et al., 2018). When targeting vigorous PA, exergames may be an appealing intervention for female players (Barr-Anderson et al., 2018), because they may internalize exergaming as PA (O'Loughlin et al., 2015; Kakinami et al., 2015). Female players also have more positive psychological responses to exergaming compared to their male counterparts (Mackintosh et al., 2016). When using exergames, females may also feel less pressured to engage in PA (George et al., 2016). PA experience has positive associations with PA participation and negative associations with sedentary gaming (Xie et al., 2017). Gaming experience may not influence physiological parameters such as energy expenditure (White et al., 2011) but may predict players' enjoyment, which itself is mediated by the satisfaction of competence needs (Kazakova et al., 2014). By increasing self-efficacy, it is plausible that players may show an interest in participating in future fitness programs (Dos Santos et al., 2016). Additionally, exhibiting a behavior in the future could partly be explained by game content (Beullens et al., 2011). Hence, exposure to environmental action cues (e.g., sport video games) may lead to increased time of exercise (Hepler et al., 2012) and including exergames in normal PA may also increase exploration intentions (Pasco et al., 2017; Joronen et al., 2017).

\section{Usability, user experience, and enjoyment}

The second aspect considers how game features might change players' PA and gaming intentions. When objectively designing better games, playtesting provides insights into players' attitudes and preferences (Phan et al., 2016). Therefore, usability and user experience tests are included in the game design process. Usability refers to the degree of user-friendliness and ease of use of a product or game. Moreover, usability is associated with usefulness and attitudes towards future use intentions (Wang and Goh, 2017; Chen et al., 2018; Li et al., 2016). A variety of different games and mechanics can also induce high intrinsic motivation, which explains their effects on positive user experience (de Vries et al., 2018). Game experience consists of several components, including flow, a state in which there is a balance between the difficulty of the task and the skills that the players possess. Video gaming is one of the best ways to induce the sense of flow (Sherry, 2006), and is positively related to physiological parameters like higher energy expenditure (Bronner et al., 2013). Game flow may increase intrinsic motivation (Plummer et al., 2017), and with the occurrence of flow, players become immersed in the game. A state of deep involvement in the game is recognized as absorption. Presence is also a psychological feeling of being in a virtual environment and shows how engaged people are while playing video games.

Another parameter that can affect playing quality is enjoyment (Howie et al., 2017). Limperos and Schmierbach (2016) evaluated how players' performance inside the game can affect enjoyment and influence players' future gameplay intentions. The researchers suggested that players with better performance experienced greater presence, which led to higher levels of enjoyment. Presence and enjoyment could mediate the relationship between player performance and future intentions to play exergames (Lee et al., 2012). Enjoyment is associated with PA, and in exergaming, enjoyment could encourage players to do movements beyond the game requirements (Lyons et al., 2014). 
There is still limited evidence on whether younger adults transfer sport and PA intentions from exergames to reality. Accordingly, the present study aimed to further understanding of how usability and playing experience contribute toward sport exergame enjoyment and future PA and sport intentions of different players. We hypothesize that female players and those with exergame and sports experience will have higher enjoyment, usability, and game experience scores. Furthermore, we hypothesize that higher usability, game experience, and enjoyment result in higher intentions for PA and sport participation.

\section{Materials and Methods}

Seventy-six healthy university students (20 female) aged $24.9 \pm 5.06$ years participated in the present study. Each participant signed an informed consent, approved by the local ethics committee and in alignment with the Declaration of Helsinki. Experienced gamers $(n=16)$ consisted of those who played exergames once a week or more, and real-swimmers $(\mathrm{n}=40)$ knew at least two swimming techniques (including front crawl) based on self-report data. Participants played different techniques (front crawl, backstroke, breaststroke, and butterfly) in a swimming exergame (Michael Phelps: Push the Limit, 505 Games, Italy) using Microsoft Xbox and Kinect for a total duration of 30 minutes. As part of the game, players also watched an ingame video tutorial on how to administer different techniques. The short version of the physical activity enjoyment scale (sPACES) was used to measure enjoyment on a 7-point Likert scale (Dunton et al., 2009). We made minor wording modifications to the items to ensure domain specificity. To illustrate, we changed some of the items to "when I was physically active playing the virtual swimming game during this study ..." and changed items to past tense (e.g., I disliked it). Both negative and positive statements were included to reduce acquiescence. The calculation of the internal consistency reliability score in this study was acceptable $(\alpha=0.76$; Nunnally and Bernstein, 1978).

We measured playability using the game experience questionnaire (GEQ) with 19 items rated on a 3-point scale (Norman, 2013). We assessed game usability using the system usability scale (SUS), which consists of ten items and is used to derive a total usability score (Jordan et al., 2014; Thirumalai et al., 2018). We modified some items to make the scale more gamespecific; namely, the word "system" was replaced with "game" and "use" with "play." To render the scores more clear and understandable, we converted SUS scores to grades using a scale defined by Bangor et al. (2009), ranging from 0 to 100. Also, overall game quality (OR) was rated by each participant on a Likert scale of 1 (worst quality) to 10 (excellent quality). The following items - "If I had a chance, I would participate in physical activity later today" and "If I had a chance to participate in physical activity, I would choose swimming." - were completed before and after participation in the present study. This allowed the research team to measure possible changes in participants' future intention to engage in PA and swimming (Dougall et al., 2011). The items were rated on a 7-point scale ranging from 1 (not likely at all) to 7 (very likely). This item has been employed in previous research exploring future intentions (Xiang et al., 2003). A single-item measure of intention has been shown to be predictive of exercise behavior measured by both fitness testing and observations of engagement during PA classes with late adolescents (Greenockle et al., 1990).

The feasibility of the questionnaires and procedures was pretested and refined with a pilot study of 10 participants who were not included in the main protocol. Upon arrival, participants completed the two questions measuring future intentions in PA and swimming. At the end of the 
gameplay, participants completed the enjoyment, usability, and game experience questionnaires. Moreover, the questions regarding PA and swimming intentions were administered again. Descriptive data (mean \pm SD) were reported for all variables, and approximate normal distribution and homogeneity of variance of independent variables (groups) were monitored. Negatively worded items of sPACES were reverse coded, and total enjoyment was recorded as the mean of the items (Ni Mhurchu et al., 2008). A two-way ANOVA with simple main effects analysis was used for enjoyment, SUS, GEQ and its components, and overall quality of the game. Gender, prior real swimming experience, and exergame experience were considered as between-subject factors. To determine the relationships between enjoyment, usability, game experience, and PA and swimming intentions, Spearman's correlation coefficients were used. Split-plot ANOVA was employed to analyze the changes in future PA and swimming intentions, incorporating both repeated measure effect (pre- and post-test) as well as between-group effect (male vs. female, swimmer vs. non-swimmer, and exergame novice vs. experienced). Statistical significance was determined at the alpha level of 0.05 , and the effect size was computed for each analysis using Hedges' $g$ to measure the practical significance of findings (Hedges and Olkin, 1985). To interpret the $g$ effect size, Cohen's guidelines (i.e., $0.2=$ small, $0.5=$ medium, and above $0.8=$ large) were employed, as well as corresponding figures for correlations (i.e., 0.1, 0.3, and 0.5; Cohen, 2013).

\section{Results}

Descriptive statistics for different variables are reported by different performing groups in Tables 1 and 2 . There was no statistically significant difference in mean usability scores, GEQ and its components, and overall quality of the game between groups and their interactions $(p>0.05$; $0.002<$ Hedges' $g<0.38$ ). Nonetheless, there was a statistically significant interaction in the effects of exergame experience on enjoyment $F(1,68)=4.918, p=0.030$, Hedges' $g=0.62$. The average SUS score was 55.34 \pm 6.35 , which is considered "OK" (Bangor et al. 2009). We also present four GEQ components in Table 2. PA intention increased for all participants $(F(1,68)=$ $\left.1.01 ; p=0.006, d_{\text {Cohen }}=0.17\right)$ but changes in swimming intentions remained similar $(p=0.32$, $d_{\text {Cohen }}=0.03$ ). Additionally, the magnitude of the main effect was not contingent on gender, real swimming experience, and exergame experience $(p>0.05)$. 
Table 1. Descriptive statistics (Mean \pm SD) for different variables by performing groups.

\begin{tabular}{|c|c|c|c|c|c|c|c|c|c|c|}
\hline & & \multicolumn{3}{|c|}{ Before } & \multicolumn{6}{|c|}{ After } \\
\hline & & Num. & PAint & SWIMint & PAint & SWIMint & ENJ & SUS & OR & GEQ \\
\hline \multirow{2}{*}{ Gen } & $\mathrm{M}$ & 56 & $4.21 \pm 2.90$ & $3.09 \pm 2.05$ & $4.43 \pm 2.82$ & $3.63 \pm 2.43$ & $4.22 \pm 0.66$ & $55.28 \pm 6.78$ & $7.80 \pm 1.44$ & $1.98 \pm 0.26$ \\
\hline & $\mathrm{F}$ & 20 & $3.25 \pm 2.55$ & $2.50 \pm 1.53$ & $3.55 \pm 2.62$ & $3.25 \pm 1.91$ & $4.39 \pm 0.41$ & $55.50 \pm 5.10$ & $8.10 \pm 1.11$ & $1.94 \pm 0.29$ \\
\hline Swir & $\mathrm{Y}$ & 40 & $4.30 \pm 2.78$ & $3.18 \pm 1.92$ & $4.00 \pm 2.71$ & $3.40 \pm 2.06$ & $4.26 \pm 0.71$ & $55.75 \pm 9.60$ & $7.87 \pm 1.14$ & $1.99 \pm 0.32$ \\
\hline Exp. & $\mathrm{N}$ & 36 & $3.58 \pm 2.88$ & $2.67 \pm 1.95$ & $4.42 \pm 2.88$ & $3.67 \pm 2.56$ & $4.27 \pm 0.48$ & $55.23 \pm 5.27$ & $7.88 \pm 1.42$ & $1.96 \pm 0.25$ \\
\hline Exer & Y & 16 & $4.56 \pm 2.73$ & $3.06 \pm 1.43$ & $5.69 \pm 1.88$ & $4.00 \pm 1.89$ & $3.91 \pm 0.91$ & $55.00 \pm 7.73$ & $7.77 \pm 1.59$ & $1.97 \pm 0.28$ \\
\hline Exp. & $\mathrm{N}$ & 60 & $3.80 \pm 2.86$ & $2.90 \pm 2.06$ & $3.80 \pm 2.86$ & $3.40 \pm 2.39$ & $4.36 \pm 0.46^{*}$ & $55.72 \pm 4.41$ & $8.00 \pm 1.06$ & $1.97 \pm 0.25$ \\
\hline Total & & 76 & $3.96 \pm 2.83$ & $2.93 \pm 1.94$ & $4.20 \pm 2.78$ & $3.53 \pm 2.30$ & $4.27 \pm 0.61$ & $55.34 \pm 6.35$ & $7.88 \pm 1.36$ & $1.97 \pm 0.27$ \\
\hline
\end{tabular}

Num.: Number; PAint: PA intention; SWIMint: Swimming intention; ENJ: Enjoyment; SUS: System usability scale score; OR: Overall rating; GEQ: Game experience questionnaire; Gen: Gender; Swim Exp.: Swimming experience; Exer Exp.: Exergame experience; M: Male; F: Female; Y: Yes; N: No. 
There were low-to-moderate correlations between overall rating and enjoyment ( $\rho=$ $0.420)$, GEQ and usability $(\rho=0.457)$, GEQ and the overall rating $(\rho=0.346)$, GEQ and postPA $(\rho=-0.232)$, absorption and usability $(\rho=0.309)$, absorption and the overall rating ( $\rho=$ $0.278)$, flow and usability $(\rho=0.302)$, flow and the overall rating $(\rho=0.486)$, presence and the overall rating $(\rho=0.267)$, immersion and usability $(\rho=0.372)$, and immersion and post-PA ( $\rho=$ $-0.235)$ and post- swimming intention $(\rho=-0.275)$, all for $p<0.05$. There was no correlation between usability and enjoyment and future intentions of either PA or real swimming $(p>0.05)$.

Table 2. GEQ components' scores (Mean \pm SD) between performing groups.

\begin{tabular}{llccccc}
\hline & & Num. & Absorption & Flow & Presence & Immersion \\
\hline Gen & $\mathrm{M}$ & 56 & $1.61 \pm 0.36$ & $1.89 \pm 0.33$ & $2.10 \pm 0.39$ & $2.31 \pm 0.52$ \\
Swim & $\mathrm{F}$ & 20 & $1.58 \pm 0.34$ & $1.95 \pm 0.28$ & $1.91 \pm 0.38$ & $2.32 \pm 0.59$ \\
Exp. & $\mathrm{N}$ & 40 & $1.62 \pm 0.36$ & $1.95 \pm 0.34$ & $2.09 \pm 0.39$ & $2.22 \pm 0.58$ \\
Exer & $\mathrm{Y}$ & 16 & $1.59 \pm 0.34$ & $1.86 \pm 0.29$ & $2.01 \pm 0.39$ & $2.41 \pm 0.47$ \\
Exp. & $\mathrm{N}$ & 60 & $1.65 \pm 0.43$ & $2.08 \pm 0.26$ & $2.16 \pm 0.39$ & $2.06 \pm 0.57$ \\
Total & & 76 & $1.60 \pm 0.33$ & $1.86 \pm 0.32$ & $2.02 \pm 0.39$ & $2.38 \pm 0.51$ \\
\hline
\end{tabular}

Num.: Number; Gen: Gender; Swim Exp.: Swimming experience; Exer Exp.: Exergame experience; M: Male; F: Female; Y: Yes; N: No.

\section{Discussion}

The aim of the present study was to compare enjoyment, usability, and gaming experience of a sport exergame in different performing groups, and to determine whether active gameplay increases players' intentions to participate in PA and the associated real sport. Our results show that psychological parameters were not different between performing groups, but players with less exergame experience enjoyed the game more. Furthermore, PA intentions increased for all participants, but swimming intentions remained unchanged.

\section{Gender, exergame, and real sport experience}

Male and female players had similar psychological parameters, which stand in opposition to the findings of Jenney et al. (2013), who attributed higher enjoyment to female players. One possibility is that swimming, as an activity, is considered neither feminine nor masculine, and therefore both genders enjoyed it equally. Contrary to previous research indicating that male players are more likely to enjoy competitive games (Soltani and Vilas-Boas, 2017), in our game, which was also competitive in nature, both genders enjoyed the game equally. It is plausible that the type of competition (competing against a computer) may not affect levels of enjoyment in either gender. Players with no prior exergame experience enjoyed the game to a greater extent than those with a history of playing exergames. This was in contrast with the findings of previous research (Depping et al., 2016; Hwang and Lu, 2018; Lai et al., 2012; Sun, 2012). The novelty of the exergame might have contributed to higher enjoyment levels (Garn et al., 2012). Experienced players and those with real swimming experience may have understood the game mechanics, and their movements may have not completely transferred to the game (Soltani et al., 2016). Accordingly, participants may have found the game to be inaccurate or unrealistic, 
compromising game enjoyment. These findings led to the rejection of the first hypothesis, that female players and those with exergame and swimming experience would have higher psychological parameters.

\section{Usability, user experience, and enjoyment}

Based on the usability score, this game was considered "OK" for products evaluated by the SUS (Bangor et al., 2009). It denoted medium perceived usability from players but was not correlated with intentions. Therefore, the second hypothesis, stating that usability is correlated with intentions, is rejected. Additionally, those who found the challenges of the game easy to handle might have rated the game usability and overall rating higher (González Sánchez et al., 2009). Our game setup and duration of gameplay (30 minutes) were compatible with the GEQ, allowing players to immerse themselves in the game. Similar to previous research, immersion was positively associated with enjoyment (Sun et al., 2015), and the GEQ was not different among performing groups (Depping et al., 2016). The performance of the game itself might have contributed to keeping the game experience similar between performing groups. Furthermore, components of the GEQ were not different between groups and were not correlated with intentions. Therefore, the second hypothesis, stating that the GEQ is correlated with intentions, is rejected.

Similar to Agarwal and Karahanna (2000), players' level of absorption to information technology is influenced by perceived usefulness and perceived ease of use. Problems with the Kinect's movement detection may have caused players to rate the absorption part of the GEQ lower. It should also be noted that absorption is a general response to any form of information technology, whereas immersion is the actual experience of players during video gaming. Nogueira et al. (2016) suggested that feedback functionality affects players' immersion, and as movement patterns of players were recognized similarly by the gaming platform, they might have immersed equally. Although presence was not different between performing groups, as real swimmers mimicked real-world swimming, they might have rated the presence component higher than non-swimmers. Similar to previous research, presence did not have any influence on future exercise intention (Kim et al., 2014). This might be because: 1) the technology did not make players think it could substitute for the real-world activity; or 2) the exposure to the virtual world was not sufficiently long enough. While immersion is considered an essential part of good user experience, it might also be affected by other parameters, such as task difficulty. González Sánchez et al. (2009) also argued that the degree to which the game contents are believable defines players' immersion. Those who thought that the game was not realistic enough or that their movements were not translated in the game correctly might have been less immersed and, therefore, believed that they needed to complete further PA and swimming. Higher scores of flow in players with exergame experience might be related to their performance and greater enjoyment of completing the task at hand (Plummer et al., 2017). Additionally, contrary to the findings of Plummer et al. (2017), gender differences in performing the task were not influenced

by flow state, which might have been caused by similar movement detection of the Kinect sensor between male and female players.

Previous exergaming experience seems to decrease levels of enjoyment. Mastery over the exergame and understanding the gaming mechanics might have contributed to this decrease (Sherry, 2006; Lai et al., 2012). On the other hand, while enjoyment may predict future exergame play (Limperos and Schmierbach, 2016), our results suggest that it may not be a 
predictor of future PA and real swimming participation. Therefore, the second hypothesis, stating that enjoyments and intentions are correlated, is rejected. It is possible that self-determined motivation to participate in PA could affect PA intentions more than a single exergame session (Standage et al., 2003). As enjoyment was reported as a crucial aspect of flow experience (Csikszentmihalyi, 2014), and with no association between these two constructs in our study, it seems that challenge and deep concentration (Klasen et al., 2012) play a vital role in characterizing flow. This was also confirmed by the correlations between immersion and usability, and between usability and flow. While it has been reported that exergames are less enjoyable than other types of video games (Lyons et al., 2011), it should be noted that removing or replacing traditional video games does not affect the enjoyment of PA (Abbott et al., 2014).

\section{$P A$ and sport intentions}

Converting sedentary screen-time to activity is a challenging endeavor. As previously argued, higher enjoyment and positive feelings of winning may contribute to the elevation of players' self-esteem, leading to more frequent participation in real sports (Adachi and Willoughby, 2013). Although changes in PA intentions increased from pre- to post-test, a close examination of the associated effect size $\left(d_{\text {Cohen }}=0.17\right)$ indicate that it might not be very meaningful. This supports the notion that PA intentions might have increased due to the novelty and fun nature of the game employed in the present study (Garn et al., 2012; Kari and Makkonen, 2014). Initial impressions of exergames may be a product of response shift (Campbell, 1957), and such brief exposure may not be an accurate impression of PA and sport behavior change.

The magnitude of the main effect changes in PA intentions was not contingent on gender, real swimming and exergame experience, which means that neither of the groups changed more. While female players are more likely to play exergames in the future (Sun, 2012), our data showed that gender might not be a predicting factor for either PA or real swimming participation intentions (Xu et al., 2016; Adachi and Willoughby, 2013). Similar to previous research, PA behaviors of experienced players were similar to novices (Barr-Anderson et al., 2018). Being active with virtual reality technologies depend on personal motivations and expectations (Marquet et al., 2017). Feelings of competence over the video game might have contributed to slightly higher PA intentions in experienced players. These players might have also understood the game mechanics and how to exert less effort. Furthermore, they might have felt that being active with the game was not enough, and therefore, they would need more PA later. Players without real swimming experience also had higher PA intention change compared to real swimmers. Competence in playing the game might have contributed to this feeling.

Swimming intentions were not different after playing the game employed in the present investigation. One explanation is that future swimming intentions of those who frequently swim may not be affected by playing active video games. Exergames may only psychologically and positively affect those who are enthusiastic about doing exercise (Huang et al., 2019). Therefore, playing a video game may not affect the intention to participate in the real sport on its own. However, the competitive nature of the game might have contributed to the higher swimming intentions of male players. Additionally, encouraging players to modify their exercise behavior has been influenced by past behavior, for example, being a real swimmer (Hepler et al., 2012). However, changes in the swimming intentions of those without real swimming experience were larger than the changes associated with real-swimmers. Non-swimmers might have thought that they were competent enough to transfer to real swimming after playing the game. While previous 
research suggests that parental support and presence of sports facilities are strongly correlated to moderate-to-vigorous PA and sedentary behaviors (Tandon et al., 2014), several other factors might explain why the presence of a sport exergame did not change swimming intentions. Game mechanics (swimming in place) and lack of forces from water might have been contradictory to the players' exercise habits and have induced an unrealistic feeling for the players (Kari and Makkonen, 2014). Pragmatic gameplay, which happens even after short exposure to the game, might have contributed to the lack of change in PA intentions. An absence of challenge and simplicity of the exergame used in the present study might have also caused participants to conclude that the game is not an adequate means of sport.

\section{Limitations and future research}

In this study, several psychological measurements were incorporated to further understanding of the enjoyment and usability aspects of sport exergaming. Our sample consisted of university students, which might enhance the internal validity of the study but may limit the generalizability of the reported results. Therefore, future studies could use larger and more balanced samples. As the SUS might be influenced by age (Quiroga et al., 2009), it is important to consider participants from different age groups and exercise backgrounds. Participants might have scored their enjoyment higher due to the novelty of the game employed in the present investigation. However, biomechanical evaluations suggest that after playing for some time, many players switch to pragmatic gameplay - that is, to exert less effort (Soltani et al., 2016). Since many exergames consist of repetitive movements, boredom might also ensue as players begin to understand the associated game mechanics. Future studies should seek to confirm whether enjoyment and adherence remain high despite a sense of mastery over the game.

While our gameplay duration was relatively long (around 30 minutes) and previous research suggests that flow can occur with a broad range of activity durations (Chen, 2007), comparisons of short-term and long-term gaming sessions are necessary to explore different players' reactions. Longitudinal studies of enjoyment are also necessary to adjust the games' difficulty. While usability measurements may not be possible in longitudinal studies, alternative methods such as ethnographic observations and qualitative user' feedback could be employed by researchers. As different games and platforms influence enjoyment differently (Thin et al., 2013), it is also important to measure these parameters on similar games across different platforms such as Nintendo Wii. Although players had the possibility to customize their avatar inside the game, which has been shown to affect exercise intention (Waddell et al., 2015), similar gaming profiles were used for all players to avoid various exertion levels. A player's competence is perceived to be higher when male avatars are used, especially for female players (Kaye et al., 2017). Although our testing sessions were held during the early hours of the day (around 9 a.m.), participants may have perceived "later today" and "future" differently. For example, participants might have responded "Not likely at all" due to other commitments in the day, because they felt that they had performed enough PA during the exergaming session. Future research could also compare the near future (later today) and further distant future by employing subtle changes to the wording of relevant measures. Future longitudinal studies should also explore if real sport and PA participation intentions lead to actual future participation. 


\section{Conclusions}

The purpose of this study was to compare usability, playability, and future intentions of participation in PA and the real sport after bouts of a swimming exergame with players of different gender, swimming experience, and exergame experience. The results indicate that a swimming exergame might be used as a tool to encourage the player to participate in PA, but this exergame does not appear to change swimming intentions. The novelty and entertainment elements of the swimming exergame may play a role in influencing players' attitudes towards PA. As realistic movements are more likely to induce presence, improving the quality of platforms' movement detection might make the gameplay more natural and increase engagement in sports exergames.

\section{Acknowledgements}

Pooya Soltani received funding from Collège de France. Authors would like to thank José Manuel Fernández Sandra, Tobias Hardardt, Tim Stokes, Soroush Soltani, Caroline Hagerman, and Jonathan Bird for proofreading the manuscript.

\section{Declaration of competing interests}

None declared. 


\section{References}

Abbott RA, Smith AJ, Howie EK, et al. (2014) Effects of home access to active videogames on child self-esteem, enjoyment of physical activity, and anxiety related to electronic games: Results from a randomized controlled trial. Games Health J 3: 260-266.

Adachi PJ and Willoughby T. (2013) More than just fun and games: The longitudinal relationships between strategic video games, self-reported problem solving skills, and academic grades. J Youth Adolesc 42: 1041-1052.

Agarwal R and Karahanna E. (2000) Time flies when you're having fun: Cognitive absorption and beliefs about information technology usage. MIS Quarterly 24: 665-694.

Arundell L, Salmon J, Veitch J, et al. (2019) The relationship between objectively measured and self-reported sedentary behaviours and social connectedness among adolescents. Int $J$ Environ Res Public Health 16: 277.

Bangor A, Kortum P and Miller J. (2009) Determining what individual SUS scores mean: Adding an adjective rating scale. Journal of Usability Studies 4: 114-123.

Baranowski T. (2017) Exergaming: Hope for future physical activity? or blight on mankind? Journal of sport and health science 6: 44-46.

Barr-Anderson DJ, Cook B, Loth K, et al. (2018) Physical activity and sociodemographic correlates of adolescent exergamers. J Adolesc Health 62: 630-632.

Benzing V and Schmidt M. (2018) Exergaming for children and adolescents: Strengths, weaknesses, opportunities and threats. J Clin Med 7.

Beullens K, Roe K and Van den Bulck J. (2011) Excellent gamer, excellent driver? The impact of adolescents' video game playing on driving behavior: a two-wave panel study. Accid Anal Prev 43: 58-65.

Biddiss E and Irwin J. (2010) Active video games to promote physical activity in children and youth: A systematic review. Arch Pediatr Adolesc Med 164: 664-672.

Bronner S, Pinsker R, Naik R, et al. (2016) Physiological and psychophysiological responses to an exer-game training protocol. Journal of Science and Medicine in Sport 19: 267-271.

Bronner S, Pinsker R and Noah JA. (2013) Energy cost and game flow of 5 exer-games in trained players. Am J Health Behav 37: 369-380.

Campbell DT. (1957) Factors relevant to the validity of experiments in social settings. Psychol Bull 54: 297-312.

Carson V, Staiano AE and Katzmarzyk PT. (2015) Physical activity, screen time, and sitting among U.S. adolescents. Pediatr Exerc Sci 27: 151-159.

Chen CK, Tsai TH, Lin YC, et al. (2018) Acceptance of different design exergames in elders. PLoS One 13: e0200185.

Chen J. (2007) Flow in games (and everything else). Communications of the ACM 50: 31-34.

Cohen J. (2013) Statistical power analysis for the behavioral sciences: Routledge.

Csikszentmihalyi M. (2014) Flow and the foundations of positive psychology, The Netherlands: Springer

de Vries AW, van Dieen JH, van den Abeele V, et al. (2018) Understanding motivations and player experiences of older adults in virtual reality training. Games Health $\mathrm{J} 7$.

Depping AE, Mandryk RL, Li C, et al. (2016) How disclosing skill assistance affects play experience in a multiplayer first-person shooter game. Proceedings of the $2016 \mathrm{CHI}$ Conference on Human Factors in Computing Systems. San Jose, California, USA: ACM, 3462-3472. 
Dixon R, Maddison R, Ni Mhurchu C, et al. (2010) Parents' and children's perceptions of active video games: A focus group study. J Child Health Care 14: 189-199.

Dos Santos H, Bredehoft MD, Gonzalez FM, et al. (2016) Exercise video games and exercise self-efficacy in children. Glob Pediatr Health 3: 1-6.

Dougall AL, Swanson JN, Grimm JR, et al. (2011) Tempering the decline in college student physical activity using informational interventions: Moderating effects of stress and stage of change. J Appl Biobehav Res 16: 16-41.

Dunton GF, Tscherne J and Rodriguez D. (2009) Factorial validity and gender invariance of the physical activity enjoyment scale (PACES) in older adolescents. Res $Q$ Exercise Sport 80: 117-121.

Farrow M, Lutteroth C, Rouse PC, et al. (2018) Virtual-reality exergaming improves performance during high-intensity interval training. Eur J Sport Sci: 1-9.

Fery YA and Ponserre S. (2001) Enhancing the control of force in putting by video game training. Ergonomics 44: 1025-1037.

Garn AC, Baker BL, Beasley EK, et al. (2012) What are the benefits of a commercial exergaming platform for college students? Examining physical activity, enjoyment, and future intentions. J Phys Act Health 9: 311-318.

George AM, Rohr LE and Byrne J. (2016) Impact of Nintendo Wii games on physical literacy in children: Motor skills, physical fitness, activity behaviors, and knowledge. Sports (Basel) 4: $1-10$.

González Sánchez JL, Padilla Zea N and Gutiérrez FL. (2009) From usability to playability: Introduction to player-centred video game development process. In: Kurosu M (ed) Human centered design. Berlin, Heidelberg: Springer Berlin Heidelberg, 65-74.

Greenockle KM, Lee AA and Lomax R. (1990) The relationship between selected student characteristics and activity patterns in a required high school physical education class. Res $Q$ Exerc Sport 61: 59-69.

Hedges LV and Olkin I. (1985) Statistical methods for meta-analysis, San Diego: Academic Press.

Hepler J, Wang W and Albarracin D. (2012) Motivating exercise: The interactive effect of general action goals and past behavior on physical activity. Motiv Emot 36: 365-370.

Howie EK, Campbell AC, Abbott RA, et al. (2017) Understanding why an active video game intervention did not improve motor skill and physical activity in children with developmental coordination disorder: A quantity or quality issue? Res Dev Disabil 60: 112.

Huang H, Nguyen HV, Cheng TCE, et al. (2019) A randomized controlled trial on the role of enthusiasm about exergames players' perceptions of exercise. Games Health J 8: 220226.

Hwang J and Lu AS. (2018) Narrative and active video game in separate and additive effects of physical activity and cognitive function among young adults. Sci Rep 8: 11020.

Jenney CT, Wilson JR, Swanson JN, et al. (2013) Exergame use as a gateway to the adoption of and adherence to sport-specific and general physical activity. J Appl Biobehav Res 18: 198-217.

Jordan PW, Thomas B, McClelland IL, et al. (2014) Usability evaluation in industry: CRC Press.

Joronen K, Aikasalo A and Suvitie A. (2017) Nonphysical effects of exergames on child and adolescent well-being: A comprehensive systematic review. Scand J Caring Sci 31: 449461. 
Kakinami L, O'Loughlin EK, Dugas EN, et al. (2015) The association between exergaming and physical activity in young adults. $J$ Phys Act Health 12: 789-793.

Kari T and Makkonen M. (2014) Explaining the usage intentions of exergames. Thirty Fifth International Conference on Information Systems. Auckland, New Zealand: Association for Information Systems (AIS).

Kaye LK, Gresty CE and Stubbs-Ennis N. (2017) Exploring stereotypical perceptions of female players in digital gaming contexts. Cyberpsychol Behav Soc Netw 20: 740-745.

Kazakova S, Cauberghe V, Pandelaere M, et al. (2014) Players' expertise and competition with others shape the satisfaction of competence needs, gaming gratifications, and contingent self-esteem in a gaming context. Cyberpsychol Behav Soc Netw 17: 26-32.

Kim SY, Prestopnik N and Biocca FA. (2014) Body in the interactive game: How interface embodiment affects physical activity and health behavior change. Comput Hum Behav 36: 376-384.

Klasen M, Weber R, Kircher TT, et al. (2012) Neural contributions to flow experience during video game playing. Soc Cogn Affect Neurosci 7: 485-495.

Kuipers DA, Terlouw G, Wartena BO, et al. (2017) The role of transfer in designing games and simulations for health: Systematic Review. JMIR Serious Games 5: e23.

Lai Y, Wang S and Yang J. (2012) An investigation of the exergames experience with flow state, enjoyment, and physical fitness. 2012 IEEE 12th International Conference on Advanced Learning Technologies. 58-60.

Lee H-G, Chung S and Lee W-H. (2012) Presence in virtual golf simulators: The effects of presence on perceived enjoyment, perceived value, and behavioral intention. New Media \& Society 15: 930-946.

Lewis BA, Napolitano MA, Buman MP, et al. (2017) Future directions in physical activity intervention research: expanding our focus to sedentary behaviors, technology, and dissemination. Journal of Behavioral Medicine 40: 112-126.

Li J, Theng YL, Cheong WL, et al. (2016) Exergames for the corporate wellness program in Singapore: An investigation of employees' acceptance via watching Kinect video. Digit Health 2: 1-8.

Limperos AM and Schmierbach M. (2016) Understanding the relationship between exergame play experiences, enjoyment, and intentions for continued play. Games Health J 5: 100107.

Lyons EJ, Tate DF, Ward DS, et al. (2011) Energy expenditure and enjoyment during video game play: Differences by game type. Med Sci Sports Exerc 43: 1987-1993.

Lyons EJ, Tate DF, Ward DS, et al. (2014) Engagement, enjoyment, and energy expenditure during active video game play. Health Psychol 33: 174-181.

Mackintosh KA, Standage M, Staiano AE, et al. (2016) Investigating the physiological and psychosocial responses of single- and dual-player exergaming in young adults. Games Health J 5: 375-381.

Marquet O, Alberico C, Adlakha D, et al. (2017) Examining motivations to play Pokemon GO and their influence on perceived outcomes and physical activity. JMIR Serious Games 5: e21.

Moholdt T, Weie S, Chorianopoulos K, et al. (2017) Exergaming can be an innovative way of enjoyable high-intensity interval training. BMJ Open Sport Exerc Med 3: e000258. 
Ni Mhurchu C, Maddison R, Jiang Y, et al. (2008) Couch potatoes to jumping beans: A pilot study of the effect of active video games on physical activity in children. International Journal of Behavioral Nutrition and Physical Activity 5: 8.

Nogueira PA, Torres V, Rodrigues R, et al. (2016) Vanishing scares: Biofeedback modulation of affective player experiences in a procedural horror game. J Multimodal User In 10: 3162.

Norman KL. (2013) GEQ (game engagement/experience questionnaire): A review of two papers. Interacting with Computers 25: 278-283.

Nunnally JC and Bernstein IH. (1978) Psychometric theory: McGraw-Hill.

O'Loughlin EK, Sabiston CM, Dugas EN, et al. (2015) The association between exercise behavior regulation and exergaming in adolescents. $J$ Phys Act Health 12: 328-334.

Owens SG, Garner JC, 3rd, Loftin JM, et al. (2011) Changes in physical activity and fitness after 3 months of home Wii Fit use. J Strength Cond Res 25: 3191-3197.

Pasco D, Roure C, Kermarrec G, et al. (2017) The effects of a bike active video game on players' physical activity and motivation. Journal of sport and health science 6: 25-32.

Pearson N, Haycraft E, Johnston JP, et al. (2017) Sedentary behaviour across the primarysecondary school transition: A systematic review. Prev Med 94: 40-47.

Pedersen SJ, Cooley PD and Cruickshank VJ. (2017) Caution regarding exergames: A skill acquisition perspective. Physical Education and Sport Pedagogy 22: 246-256.

Phan MH, Keebler JR and Chaparro BS. (2016) The development and validation of the Game User Experience Satisfaction Scale (GUESS). Hum Factors 58: 1217-1247.

Plummer JP, Schuster D and Keebler JR. (2017) The effects of gender, flow and video game experience on combat identification training. Ergonomics 60: 1101-1111.

Quan M, Pope Z and Gao Z. (2018) Examining young children's physical activity and sedentary behaviors in an exergaming program using accelerometry. J Clin Med 7.

Quiroga MA, Herranz M, Gómez-Abad M, et al. (2009) Video-games: Do they require general intelligence? Comput Educ 53: 414-418.

Rhodes RE, Kaos MD, Beauchamp MR, et al. (2018) Effects of home-based exergaming on child social cognition and subsequent prediction of behavior. Scand J Med Sci Sports 28: 2234-2242.

Schneekloth B and Brown GA. (2018) Comparison of physical activity during zumba with a human or video game instructor. Int J Exerc Sci 11: 1019-1030.

Schwarz A, DeSmet A, Cardon G, et al. (2018) Mobile exergaming in adolescents' everyday lifecontextual design of where, when, with whom, and how: The SmartLife case. Int J Environ Res Public Health 15.

Sherry JL. (2006) Flow and media enjoyment. Communication theory 14: 328-347.

Soltani P, Figueiredo P, Fernandes RJ, et al. (2016) Do player performance, real sport experience, and gender affect movement patterns during equivalent exergame? Comput Hum Behav 63: 1-8.

Soltani P, Figueiredo P, Ribeiro J, et al. (2017) Physiological demands of a swimming-based video game: Influence of gender, swimming background, and exergame experience. Scientific Reports 7: 5247.

Soltani P and Vilas-Boas JP. (2017) Sport exergames for physical education. In: Khosrow-Pour M (ed) Encyclopedia of Information Science and Technology, Fourth Edition. Hershey, PA, USA: IGI Global, 7358-7367. 
Staiano AE, Beyl RA, Guan W, et al. (2018) Home-based exergaming among children with overweight and obesity: A randomized clinical trial. Pediatr Obes 13: 724-733.

Staiano AE and Calvert SL. (2011) Exergames for physical education courses: Physical, social, and cognitive benefits. Child Development Perspectives 5: 93-98.

Standage M, Duda JL and Ntoumanis N. (2003) A model of contextual motivation in physical education: Using constructs from self-determination and achievement goal theories to predict physical activity intentions. Journal of Educational Psychology 95: 97-110.

Straatmann VS, Almquist YB, Oliveira AJ, et al. (2019) Stability and bidirectional relationship between physical activity and sedentary behaviours in Brazilian adolescents: Longitudinal findings from a school cohort study. PLoS One 14: e0211470.

Sun H. (2012) Exergaming impact on physical activity and interest in elementary school children. Res $Q$ Exerc Sport 83: 212-220.

Sun H, Li S, Zhu Y, et al. (2015) The effect of user's perceived presence and promotion focus on usability for interacting in virtual environments. Appl Ergon 50: 126-132.

Tandon P, Grow HM, Couch S, et al. (2014) Physical and social home environment in relation to children's overall and home-based physical activity and sedentary time. Prev Med 66: 3944.

Thin AG, Brown C and Meenan P. (2013) User experiences while playing dance-based exergames and the influence of different body motion sensing technologies. International Journal of Computer Games Technology 2013: 1-7.

Thirumalai M, Kirkland WB, Misko SR, et al. (2018) Adapting the Wii Fit balance board to enable active video game play by wheelchair users: User-centered design and usability evaluation. JMIR Rehabil Assist Technol 5: e2.

Waddell TF, Sundar SS and Auriemma J. (2015) Can customizing an avatar motivate exercise intentions and health behaviors among those with low health ideals? Cyberpsychol Behav Soc Netw 18: 687-690.

Wang X and Goh DH. (2017) Video game acceptance: A meta-analysis of the extended technology acceptance model. Cyberpsychol Behav Soc Netw 20: 662-671.

White K, Schofield G and Kilding AE. (2011) Energy expended by boys playing active video games. J Sci Med Sport 14: 130-134.

Wu Z, Li J and Theng YL. (2015) Examining the influencing factors of exercise intention among older adults: A controlled study between exergame and traditional exercise. Cyberpsychol Behav Soc Netw 18: 521-527.

Xiang P, McBride R, Guan J, et al. (2003) Children's motivation in elementary physical education: An expectancy-value model of achievement choice. Res Q Exerc Sport 74: 2535.

Xie H, Scott JL and Caldwell LL. (2017) Urban adolescents' physical activity experience, physical activity levels, and use of screen-based media during leisure time: A structural model. Front Psychol 8: 2317.

$\mathrm{Xu} \mathrm{X}$, Theng Y, Li J, et al. (2016) Investigating effects of exergames on exercise intentions among young-old and old-old. Proceedings of the 2016 CHI Conference Extended Abstracts on Human Factors in Computing Systems. San Jose, California, USA: ACM, 2961-2968. 